\title{
Multi-objective Optimal Design of a Toroidally Wound Radial-flux Halbach Permanent Magnet Array Limited Angle Torque Motor
}

\author{
Shuai Wu, Member, IEEE, Xiangyu Zhao, Zongxia Jiao, Member, IEEE, \\ Patrick Chi-kwong Luk,Member, IEEE, and Chenxiao Jiu
}

\begin{abstract}
This paper presents the modeling, optimization and validation of a toroidally wound radial-flux Halbach array permanent magnet limited angle torque motor. A fully parameterized and flexible equivalent magnetic circuit model of the proposed motor, which is arranged in matrix form by means of Kirchhoff's current laws for computational efficiency and ease of extension, is introduced for preliminary design. To optimize the design, a multi-objective optimization process is carried out and the multi-objective particle swarm optimization method is used to obtain the Pareto front of the desired objectives. An approved solution in Pareto front is selected and validated by finite element analysis method. A prototype based on the final design is built and tested. The experiment results further underpin the effectiveness of the proposed design and optimization approach.
\end{abstract}

Index Terms-Brushless motor, direct current motors, finite element analysis, Halbach array, limited angle torque motor, magnetic circuit, multiobjective optimization, Pareto optimization

\section{NOMENCLATURE}

$\alpha_{c} \quad$ Stator winding arc angle, see Fig. 2.

$\alpha_{m} \quad$ PM arc angle of pole, see Fig.2.

$\alpha_{0} \quad$ Arc angle between two coils, see Fig.2.

$\alpha_{1} \quad$ Woring range, see Fig.2.

$\boldsymbol{G} \quad$ Permeance matrix.

$\mu \quad$ Relative permeability.

$\phi, \phi \quad$ Magnetic flux and its vector form.

$B \quad$ Magnetic flux density.

$d_{c} \quad$ Diameters of conductor.

$F, \boldsymbol{F}$ Absolute magneto-motive force and its vector form. $k_{1}, k_{2}, k_{3}$ The constants of B-H curve of different materials. $K_{e}, K_{t}$ Back-emf coefficient and torque constant of motor, respectively.

Manuscript received Month $x x, 2 x x x$; revised Month $x x, x x x x$; accepted Month $\mathrm{x}, \mathrm{xxxx}$.

This work is supported by National Aviation Science Foundation under Grant (No. 20130751013) and National Key Basic Research and Development Program under Grant (2014CB046401) and National Science and Technology Major Project under Grant (2014ZX04001-141).

Shuai Wu, Xiangyu Zhao and Zongxia Jiao are with the School of Automation Science and Electrical Engineering, Beihang University, Beijing, 100191 China; (e-mail: wushuai.vip@gmail.com).

Patrick Chi-kwong Luk is with Electric Power and Drives Group, Power Engineering Centre, Cranfield University, Cranfield, MK43 OAL, U.K.; (email: p.c.k.luk@cranfield.ac.uk).

Chenxiao Jiu is with Xi'an flight automatic control research institute.

\author{
$L_{m} \quad$ Axis length of motor. \\ $L_{w} \quad$ Winding Layers. \\ $N \quad$ The total count of sectors. \\ $N_{p} \quad$ The pole count of motor. \\ $N_{e} \quad$ The count of sectors of gap between neighbor coils. \\ $N_{m} \quad$ The count of sectors of radial-magnetized PM. \\ $N_{w} \quad$ The count of sectors of working range. \\ $R \quad$ Equivalent magnetic reluctance. \\ $r_{c o} \quad$ Outer radius of motor, see Fig.2. \\ $R_{c}, L_{c}$ Resistance and inductance of motor, respectively. \\ $r_{r i} \quad$ Inner radius of motor, see Fig.2. \\ $T$ Torque of motor. \\ $t_{h} \quad$ Thickness of adjacent PM, see Fig.2. \\ $t_{m} \quad$ Thickness of main PM, see Fig.2. \\ $t_{r} \quad$ Thickness of rotor, see Fig.2. \\ $t_{s} \quad$ Thickness of stator, see Fig.2.
}

\section{INTRODUCTION}

$\mathbf{T}$ HE limited angle torque motor (LATM) is an electromechanical actuator which can only rotate within a limited angular range. It is widely used in swing servo control, such as in hydraulic rotary servo valves. When used in rotary servo valve application, the LATM usually requires high control precision and quick response within a small angular range.

Therefore, the conventional alternating current (AC) motor or brush-less permanent magnet (BLPM) motor is not suitable due to the inherent cogging torque and low position precision.

Various types of LATM have been reported in the literature [1]. The LATM with the configuration of a toroidal wound stator and a permanent-magnet rotor has attracted continuous attention for its advantages of accurate positioning capability, low cogging torque, and high reliability [2]-[5]. This type of toroidally wound LATM does not have the cogging problem due to the constant reluctance path and relatively large airgap [2]. Therefore, optimized design methods such as using Halbach magnetization to strengthen the magnetic field and compensate for the reduced torque are desirable [6], [7].

The optimal design of the motor can be done in several ways. The finite element analysis (FEA) method can directly calculate the flux pattern with high accuracy, and is usually applied in the detailed design phase for specification purposes, such as rotor shape optimization [8], and torque 
ripple reduction [9]. But the entire process of FEA is usually computationally expensive. Moreover, changing the design parameters in FEA, especially the structure parameters (e.g. pole number, winding arrangement) in the preliminary design phase, often requires the model to be reconstructed. On the other hand, an analytical approach can allow the designer to scan the full design space to optimize the parameters with respect to the given requirements and constraints [10], [11]. The equivalent magnetic circuit (EMC) model is a widely used technique and often serves as the first step in the analysis and design of electric machines [12]-[15]. In EMC, magnetic reluctance is analogous to resistance, flux is analogous to current, and the excitation of magnetic circuits is analogous to voltage. Therefore, the magnetic field characteristics can be obtained using electric circuit principle, such as Kirchhoff's Current Laws (KCL) [16]. The EMC model is usually only employed for preliminary design, and is often incorporated with other methods such as FEA for further confirmation of the results [17].

The optimization of LATM needs to improve several performances simultaneously, such as higher torque constant, shorter response time, smaller size and weight, and higher linearity. However, these objectives usually conflict with each other. Thus the multi-objective optimization (MOO) method in which conflicting objectives are being considered simultaneously is required. For MOO problems, using a set of weighted coefficients to trade off objectives is a practical way [18]. But the more advanced ones often involve artificial intelligence methods to obtain the Pareto front of the objectives under the design constraints [19]-[21].

This study aims at developing a LATM for hydraulic rotary direct drive servo valve. The major specifications include: torque constant bigger than $0.5 \mathrm{Nm} / \mathrm{A}$, high torque linearity in the range of $\pm 10^{\circ}$, and step response time less than $10 \mathrm{~ms}$. A toroidally wound radial-flux Halbach permanent magnet array type LATM is proposed. This paper presents the modeling, optimization, prototyping and testing of the motor. A fully parameterized and flexible EMC model of the proposed motor, which is arranged in KCL matrix format for computational efficiency and ease of extension, is first discussed. Based on the EMC model, a MOO process is carried out to obtain the Pareto front of the desired objectives. Then an approved solution in Pareto front is validated by FEA method and further optimizations are undertaken in details. Finally, a prototype of the proposed LATM is manufactured and tested. The measured results verify the effectiveness and validity of the proposed design and optimization approach, and show the performances satisfy the application requirements.

\section{THE DESIGN OF PROPOSED LATM}

The structure of proposed LATM is shown in Fig. 1. It is a toroidally wound type solid core stator and a rareearth permanent magnetic (PM) pole-tip rotor. It suits for high precision servo control because it is no cogging torque in slot-less structure. The rotor carries several main PMs which are radially magnetized to form the magnetic poles, and neighboring magnetic poles which are magnetized in the opposite directions to form a closed flux circuit. Between two neighboring PMs, there is an adjacent PM magnetized circumferentially to form a Halbach array in order to enhance the flux density in the air-gap. The coils wound on the stator cover the range of the main PMs. The interaction of the two magnetic fields of the PM and the excited winding produces an electromagnetic toque. The magnitude and direction of armature current determine the magnitude and direction of the generated electromagnetic torque and hence the rotor direction. Fig. 1 shows the 4-pole design but the structure and principle are similar for motors with different pole number. The geometry definitions of one pole of the 4-pole LATM are shown in Fig. 2. And the theoretical torque versus angular position characteristic of the proposed design is shown in Fig. 3. It indicates that the $\alpha_{1}$ is equal to $0.5\left(\alpha_{c}-\alpha_{m}\right)$.

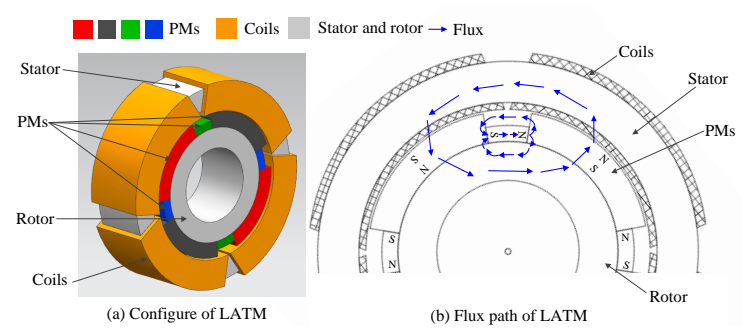

Fig. 1. Structure of LATM. (a) Configure of 4-pole design, (b) Magnetized direction and flux path.

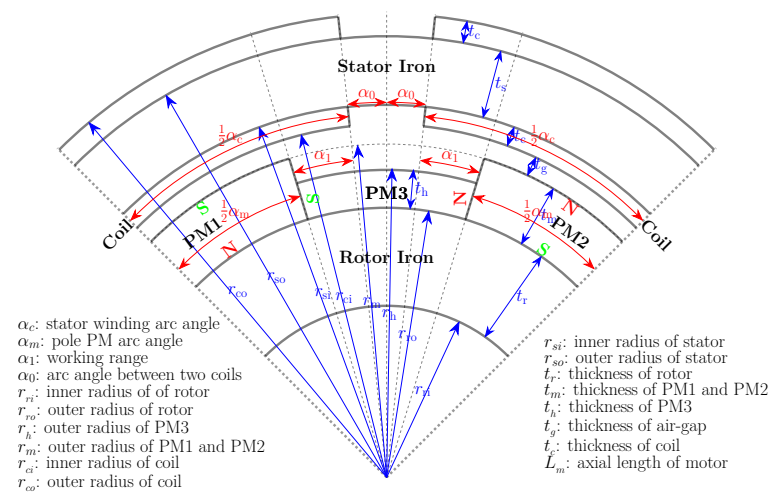

Fig. 2. Geometry definitions of LATM.

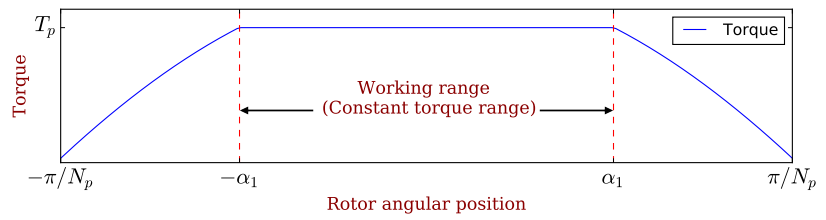

Fig. 3. Torque-position characteristic of proposed LATM.

\section{Analytical Modeling by EMC}

\section{A. Geometry definitions and EMC modeling}

The EMC model of the proposed 4-pole LATM is shown in Fig. 4. Only a quarter of motor is modeled necessarily due 


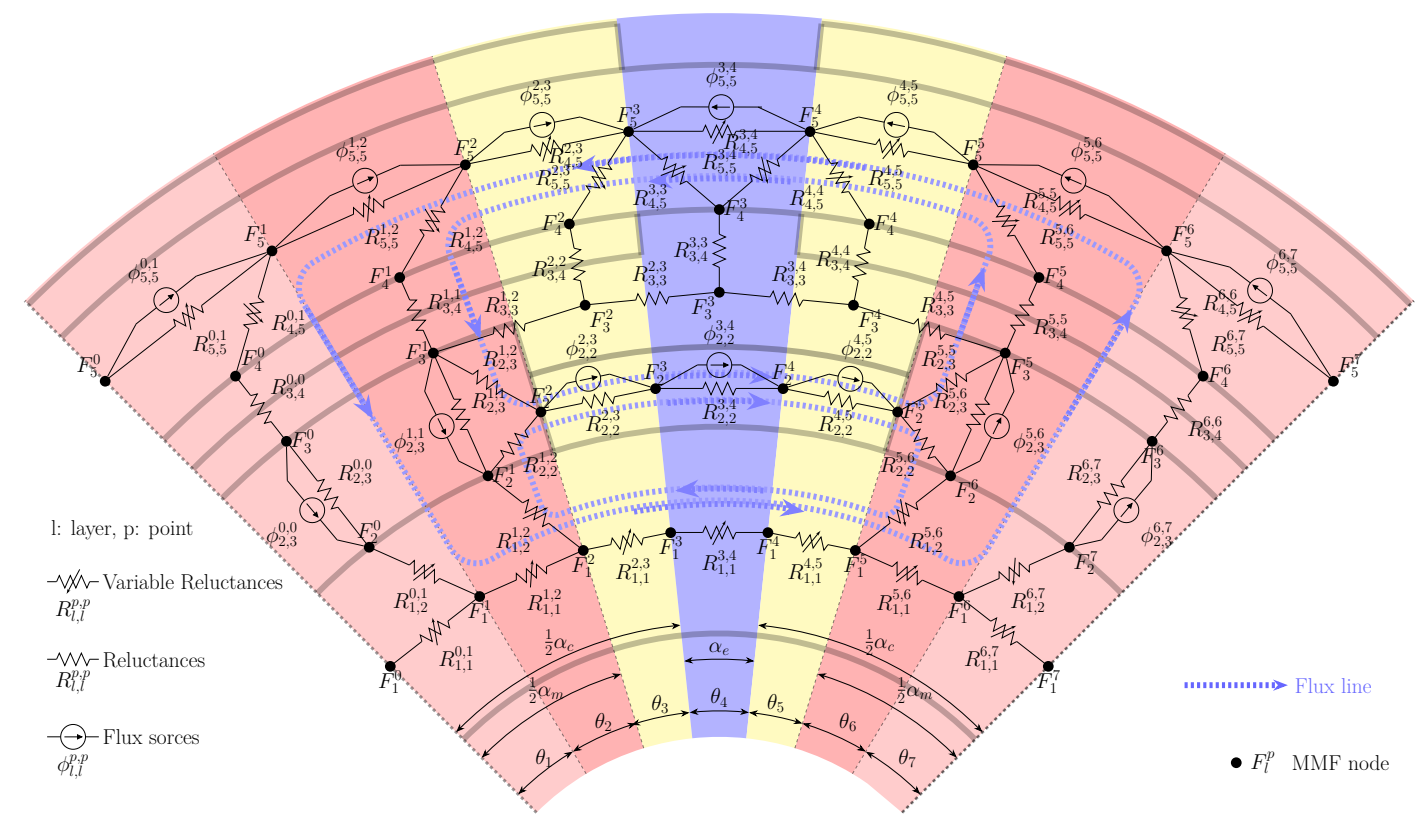

Fig. 4. Equivalent magnetic circuit model of a 4-pole design LATM. Only a quarter of the motor is modeled due to its symmetric.

to symmetry for each pole. The definitions of subscript and superscript of these symbols are list below.

$F_{l}^{p} \quad$ The MMF at different layer and node, the $(l)$ indicates the layer, and the $p$ indicates the node.

$R_{l, l}^{p, p}$ The reluctance between two nodes, the $(l, l)$ and $(p, p)$ indicate the layers and the nodes of the two ends of the reluctance, respectively.

$\phi_{l, l}^{p, p} \quad$ The flux source between two nodes, the $(l, l)$ and $(p, p)$ indicate the layers and nodes of two ends of the flux source, respectively.

The accuracy of EMC model depends on the accuracy of the model of the individual components and the coupling between the active components (e.g. magnets and windings) and passive components (e.g. air gap and iron core) [16]. This requires revisiting the fundamental principles in magnetic circuits. Dividing the flux paths into several paths and regions can make the model flexible and get reasonably good overall results. Fig. 4 shows the EMC model of a quarter of the 4pole LATM. It is divided into 4 layers in the radial direction and 3 regions in the circumferential direction. The 4 layers in radial direction include the rotor, PMs, air-gap and stator. The reluctances in the rotor and stator are considered variable with flux density. They are divided into two types. One is along the circumferential direction, and the other is between the iron and air-gap or PM. The magnetic reluctances are calculated using the geometrical dimensions and magnetic permeability. The reluctances along circumferential direction are simplified to a cuboid and the reluctances between iron and air-gap or PM are regarded as a quarter cylinder. The details of these two type reluctances are as shown in Table. II. The EMC model is divided into three regions in circumferential direction which are presented in different colors in Fig. 4. The gap between two neighboring coils is colored in blue, the working ranges in yellow, and the regions of radial-magnetized PMs in red. The number of sectors of these three regions can be modified to trade off accuracy and computation cost. More sectors can improve the accuracy because each sector of the iron has own relative permeability according to its flux density. But the computation cost is square to the sector count. The total count of sector count of one pole equals $N=\left(N_{m}+N_{w}+N_{e}\right)$.

Five layers of MMF nodes are employed for the proposed EMC model as shown in Fig. 4. The first layer MMF nodes are set at the middle of the rotor. The second layer MMF nodes are set on the junction surface between the rotor and radial-magnetized PMs and the middle of the circumferentialmagnetized PM. The third layer MMF nodes are set on the junction surface between PM and air-gap. The forth layer MMF nodes are set on the junction surface between the air-gap and stator. The fifth layer MMF nodes are set at the middle of the stator. The reluctances and equivalent flux sources are defined between these MMF nodes. The count of MMF nodes depends on divided sectors of each region. The EMC model has a total of $5 N+3 \mathrm{MMF}$ nodes. For example, in Fig. 4, $N_{m}=4, N_{w}=2$, and $N_{e}=1$, and $N=7$, the count of MMF nodes of five layer are $N+1=8, N+1=8, N=7$, $N=7$ and $N+1=8$, respectively.

In this paper, the EMC model is expressed in matrix which can be readily solved by KCL method which is presented as

$$
G \boldsymbol{F}=\phi .
$$

The $\boldsymbol{G}, \boldsymbol{F}$ and $\phi$ can be presented as follow and the details are given in the Appendix.

$$
\boldsymbol{G}=\left[\begin{array}{ccccc}
\boldsymbol{G}_{11} & \boldsymbol{G}_{12} & 0 & 0 & 0 \\
\boldsymbol{G}_{21} & \boldsymbol{G}_{22} & \boldsymbol{G}_{23} & 0 & 0 \\
0 & \boldsymbol{G}_{32} & \boldsymbol{G}_{33} & \boldsymbol{G}_{34} & 0 \\
0 & 0 & \boldsymbol{G}_{43} & \boldsymbol{G}_{44} & \boldsymbol{G}_{45} \\
0 & 0 & 0 & \boldsymbol{G}_{54} & \boldsymbol{G}_{55}
\end{array}\right]_{(5 N+3) \times(5 N+3)}
$$




$$
\begin{aligned}
& \boldsymbol{F}=\left[\begin{array}{l}
\left(\boldsymbol{F}_{1}^{n}\right)_{(N+1) \times 1} \\
\left(\boldsymbol{F}_{2}^{n}\right)_{(N+1) \times 1} \\
\left(\boldsymbol{F}_{3}^{n}\right)_{N \times 1} \\
\left(\boldsymbol{F}_{4}^{n}\right)_{N \times 1} \\
\left(\boldsymbol{F}_{5}^{n}\right)_{(N+1) \times 1}
\end{array}\right]_{(5 N+3) \times 1} \\
& \boldsymbol{\phi}=\left[\begin{array}{l}
\left(\boldsymbol{\phi}_{1}^{n}\right)_{(N+1) \times 1} \\
\left(\boldsymbol{\phi}_{2}^{n}\right)_{(N+1) \times 1} \\
\left(\boldsymbol{\phi}_{3}^{n}\right)_{N \times 1} \\
\left(\boldsymbol{\phi}_{4}^{n}\right)_{N \times 1} \\
\left(\boldsymbol{\phi}_{5}^{n}\right)_{(N+1) \times 1}
\end{array}\right]_{(5 N+3) \times 1}
\end{aligned}
$$

The vector of $\boldsymbol{F}$ can be obtained by product $\boldsymbol{G}^{-1}$ at each side of Eq. 1. Then the flux between neighboring MMF nodes can be calculated by the differential MMF over the reluctance between them, and the flux density can be obtained by flux over the effective area.

Since the current models only involve one pole of the motor, it can be extended to different poles design without rebuilding the network by simply adjusting the pole count and the angle of one pole. The modeling accuracy and the computational cost can also be traded off by changing the parameters of $N_{m}$, $N_{w}$ and $N_{e}$. Therefore, it is a highly flexible parametrized modeling approach for this type of LATMs and is particularly suitable for the preliminary design phase.

\section{B. Nonlinear Material Consideration}

The stator and rotor are made by ferromagnetic material with nonlinear B-H relationship. The permeability can vary significantly with the flux density. Therefore, the nonlinear material characteristic should be considered for high fidelity modeling. The relationship between the relative permeability and magnetic flux density can be modeled by the following function [17]:

$$
\mu=B /\left(k_{1} e^{k_{2} B^{2}}+k_{3}\right)
$$

These constants of the material used in the present study are $k_{1}=2.6, k_{2}=2.72$ and $k_{3}=154.4$. The $\mu$-B curve is shown in Fig. 5.

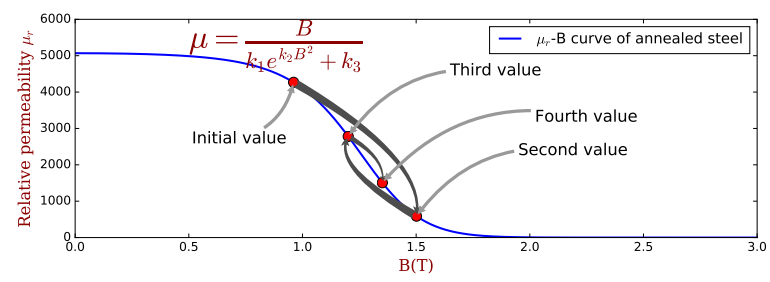

Fig. 5. Relative permeability curve vs flux density of steel.

The reluctances in the EMC model are updated by an iterative process which is shown in Fig. 5. Firstly, a reasonable permeability value, for example 4000 , is given to each sector of the stator and rotor to initiate the calculation. The flux density of each sector can be obtained and then the new relative permeability of each sector becomes:

$$
\mu_{k+1}^{n}=B_{k}^{n} /\left(k_{1} e^{k_{2}\left(B_{k}^{n}\right)^{2}}+k_{3}\right)
$$

Then the $\mu_{k+1}^{n}$ is fed back to the model to replace the previous permeability values. The process is iterated until the results are converged. The stop condition can be defined by the amount of variation of the flux density in air gap, stator and rotor between two consecutive iterations, $\max \left(\left|B_{k+1}^{n}-B_{k}^{n}\right|\right)<\epsilon$, where $\epsilon$ is a small value which can denote the relative permeability is converged. In the present study, $\epsilon=0.01 \mathrm{~T}$ is set.

\section{Validation by FEA}

The EMC model is validated by FEA method. Two group design parameters listed in Table. I are validated. The flux density distribution results of the FEA model for these two designs are shown in Fig. 6. The results of flux density in the air-gap which are calculated by FEA and EMC methods are shown in Fig. 7. The torque-angle and torque-current characteristics are shown in Fig. 8. Both the air-gap flux density and torque characteristic comparisons of the FEA and EMC method indicate that the accuracy of the proposed EMC model is entirely acceptable for preliminary design phase.

TABLE I

DESIGN PARAMETERS FOR VALIDATION

\begin{tabular}{llrr}
\hline Parameters (Units) & Symbols & Design 1 & Design 2 \\
\hline Working range & $\alpha_{1}$ & 10 & 5 \\
Pole count & $N_{p}$ & 4 & 6 \\
Thickness of rotor (mm) & $t_{r}$ & 7.5 & 7 \\
Thickness of main PM (mm) & $t_{m}$ & 7.8 & 8.8 \\
Thickness of stator (mm) & $t_{s}$ & 8 & 7.5 \\
Thickness of adjacent PM (mm) & $t_{h}$ & 5 & 5.5 \\
Diameters of conductor $(\mathrm{mm})$ & $d_{c}$ & 0.5 & 0.5 \\
Winding Layers & $L_{w}$ & 4 & 4 \\
\hline
\end{tabular}

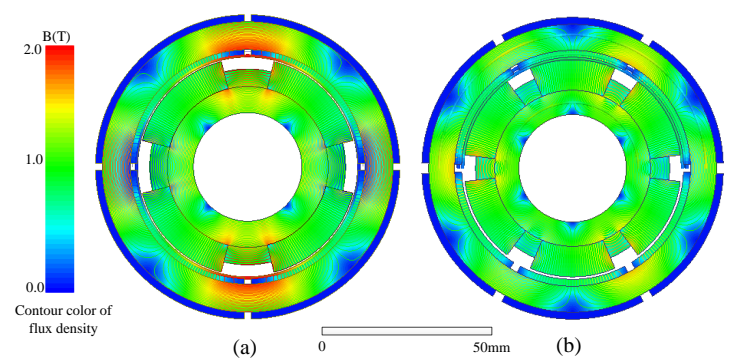

Fig. 6. Flux density distribution of FEA. (a) Desing 1. (b)Design 2.

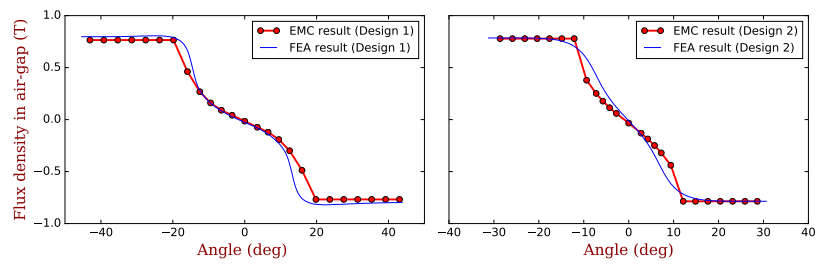

Fig. 7. Comparison of flux density in air-gap of EMC and FEA

\section{Multi-objectives Optimization Design of LATM}

\section{A. Optimization Objectives}

For the rotary servo valve application, the given requirements include the torque constant, working range, dynamic response time and linearity. Therefore, the optimization objectives considered in the present study are listed as follows. 

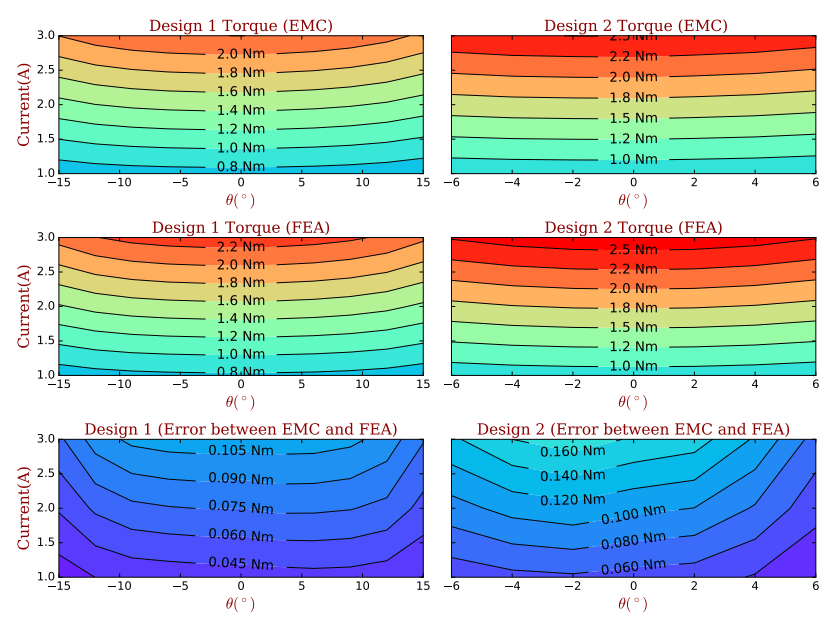

Fig. 8. Comparison of calculated torque of EMC and FEA

- Torque constant $K_{t}$. The $K_{t}$ is the basic requirement which is decided by the application.

- Dynamic response time $\tau_{t}$. The dynamic response time is important for servo applications. The simplified transfer function between input voltage $(U)$ and motor speed $(\omega)$ is a second order system $\omega(s) / U(s)=K /\left(\tau^{2} s^{2}+2 \zeta \tau s+\right.$ $1)$, where the steady-stage gain $K=1 / K_{e}$, the characteristic time $\tau=\sqrt{(L J) /\left(K_{t} K_{e}\right)}$, and the damping ratio $\zeta=\left(R_{c} / 2 L_{c}\right) \tau$. The dynamic response time should be assessed under two conditions that are distinguished by $\zeta$. (1) Over-damping $(\zeta>1)$. The second order system can be regard as two first order terms series as $1 /\left(\left(\tau_{1} s+1\right)\left(\tau_{2} s+1\right)\right)$, where $\tau_{1,2}=\tau /\left(\zeta \pm \sqrt{\zeta^{2}-1}\right)$ are two first order time constants. The total response time can be presented as $\tau_{\mathrm{t}}=\tau_{1}+\tau_{2}$. (2) Under-damping $(\zeta<1)$. Then the time constant is usually defined as the real part of the roots of the characteristic equations, $\tau / \zeta$, but increasing the imaginary part can also shorten the response time, and it will introduce some undesirable overshoot. Nonetheless, if the damping ratio is limited in an acceptable range, for example, $0.7<\zeta<1$, a quicker response can be achieved with an acceptable magnitude of overshoot, then it is preferred for servo applications. Therefore, in this condition, the total time delay can be defined as $\tau_{t}=1 /\left(\left|\vec{p}_{1}\right|+\left|\vec{p}_{2}\right|\right)=2 \tau$, where $\vec{p}_{1}$ and $\vec{p}_{2}$ are the two eigenvectors. Defining $\tau_{t}=\infty$ when $\zeta \leq 0.7$ can make the underdamping solution unacceptable. In summary, the dynamic response time can be defined as:

$$
\tau_{t}=\left\{\begin{array}{l}
\tau /\left(\zeta-\sqrt{\zeta^{2}-1}\right)+\tau /\left(\zeta+\sqrt{\zeta^{2}-1}\right), \zeta \geq 1 \\
2 \tau, \quad 1>\zeta>0.7 \\
\infty, \quad 0.7 \geq \zeta
\end{array}\right.
$$

- Torque linearity characteristic. The torque linearity has two aspects, the torque versus current and angular position. For the proposed structure, torque proportional to the current is easy to achieve when the motor is not saturated. The avoidance of saturation has been considered in the optimization process and the design which causes saturation will be excluded. Then the linearity with angular position should be particularly focused on the design parameter of $t_{h}$. Increasing $t_{h}$ can enhance $B_{g}$, which in turn increases $K_{t}$. But it also enlarges the torque differential in the working range. The simulation results by FEA in Fig. 9 illustrate this trend.

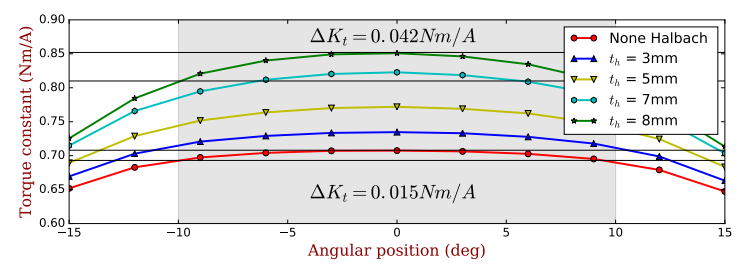

Fig. 9. Torque in the angle range with different $t_{h}$

The final objectives functions are expressed as follows:

$$
\text { Objs }=\left[\mathrm{Obj}_{1}, \mathrm{Obj}_{2}, \mathrm{Obj}_{3}\right]=\left[0.5 / K_{t}, 10 \Delta K_{t}^{\max }, 100 \tau_{t}\right]
$$

where the $\Delta K_{t}^{\max }$ is the maximum variation of torque constant during the required working range. The production constants are normalizing factors.

\section{B. Optimization parameters choice}

There are 8 parameters need to be decided, which include $N_{p}, r_{r i}, t_{r}, t_{m}, t_{h}, d_{c}, L_{w}$ and $L_{m}$. The pole count $N_{p}$ should conform to the desired working range $\alpha_{1}$. Low pole number design is suitable for a large working range. Otherwise, more poles design for large working range will make the size of PM very small, because $\alpha_{m}=2 \pi / N_{p}-\alpha_{e}-2 \alpha_{1}$, it will result in a small $K_{t}$. On the contrary, high pole number design is suitable for small working range applications. Because it reduce the total flux of each magnetic flux loop. Thus, high pole number design for small working range application helps to avoid magnetic saturation of stator and rotor. Therefore, it is good for increasing air-gap flux density which can enhance $K_{t}$. The $t_{m}$ is the primary determinant of the air gap flux density. Increasing $t_{m}$ could enlarge the air-gap flux density but the increase in gradient gets smaller. Moreover, the mass and inertia increase linearly and squarely, respectively. Thus, $t_{m}$ should be optimized to maximum overall performance. Both $t_{r}$ and $t_{s}$ influence the flux density in the rotor and stator. They should be optimized to make the flux density in the stator and rotor close to the saturation value to maximize material usage. The $d_{c}$ and $L_{w}$ decide the thickness of air-gap and resistance of the coil. The thickness of the winding $t_{c}$ equals to $L_{w} \times d_{c}$.

In the present study, the required working range is $\pm 10^{\circ}$, and several design specifications have predetermined parts of parameters which are listed below: $\alpha_{0}=2^{\circ}, r_{r i}=15.5 \mathrm{~mm}$, $r_{c o}=43.5 \mathrm{~mm}, L_{m}=25 \mathrm{~mm}$. The remaining parameters need to be optimized are $N_{p}, t_{s}, t_{m}, t_{h}, d_{c}$ and $L_{w}$.

\section{MOO problem description and method}

The MOO problem can be defined as: let $S \in R^{n}$ be an $n$ dimensional search space, and $f_{i}(\boldsymbol{x})$, be $k$ objective functions, $g_{j}(\boldsymbol{x})$, be $m$ inequality constraints, defined over $S$. Let $\boldsymbol{f}$ be a vector function defined as

$$
\begin{gathered}
\boldsymbol{f}(\boldsymbol{x})=\left[f_{1}(\boldsymbol{x}), f_{2}(\boldsymbol{x}), \cdots, f_{i}(\boldsymbol{x}], \quad i=1, \cdots, k\right. \\
\text { where } g_{j}(\boldsymbol{x}) \leq 0, \quad j=1, \cdots, m,
\end{gathered}
$$


Then, it is interesting to find a solution, $\boldsymbol{x}=\left(x_{1}, \cdots, x_{n}\right)$, that minimizes $\boldsymbol{f}(\boldsymbol{x})$. The objective functions $f_{i}(\boldsymbol{x})$ may conflict with each other, thereby, rendering it is impossible to detect a single global minimum at the same point in $S$. Usually, a solution $\boldsymbol{x}$, is said to be Pareto optimal, if and only if there is no other solution, $\boldsymbol{y}$, in $S$ such that $\boldsymbol{f}(\boldsymbol{y})$ dominates $\boldsymbol{f}(\boldsymbol{x})$. The set of all Pareto optimal solutions is called the Pareto front.

There are various methods for the MOO problem, such as multi-objective particle swarm optimization (MOPSO) method [22], Pareto-frontier differential evolution (PDE) approach [23], and fast non-dominated sorting genetic algorithm (NSGA-2) method [24].

The MOPSO method is adopted for its good global searching capability and ease of application. The pseudo code of MOPSO in the present study can be described as follows:

1) Initialize the population $S^{\mathrm{POP}}$ with the random design parameters in the defined range.

2) Evaluate each of the particles in $S^{\mathrm{POP}}$, get the performances by EMC model.

3) Store the positions (design parameters) which get the nondominant performances into the repository $S^{\mathrm{REP}}$.

4) Restore the repository to make it well-distributed, keep the size of repository under the defined capacity.

5) Compute the update speed of each particle using Eq. (10).

$$
\begin{aligned}
v_{i}(t+1)=w v_{i}(t) & +c_{1} r_{1}\left(p_{i}^{\mathrm{BEST}}(t)-x_{i}(t)\right) \\
& +c_{2} r_{2}\left(R_{h}(t)-x_{i}(t)\right),
\end{aligned}
$$

where $p_{i}^{\mathrm{BEST}}$ is its best position in searching history and $R_{h}$ is the selected leader from the repository, $w$ is the inertia coefficient of velocity; $c_{1}$ and $c_{2}$ are local and social coefficients, respectively; $r_{1}$ and $r_{2}$ are two random numbers in range $[0,1]$.

6) Compute the new position of the particles by adding the speed produced from the previous step, $S_{i}^{\mathrm{POP}}(t+1)=$ $S_{i}^{\mathrm{POP}}(t)+v_{i}(t)$, and get new positions and return back to the Step 2 until maximum iteration cycle is reached.

\section{Optimization results}

The EMC modeling and MOPSO optimization is done by Python. The sector divisions in EMC model are $N_{m}=8$, $N_{w}=4$, and $N_{e}=2$. The maximum optimization iteration of MOPSO is 100 , the particle count and the capacity of the repository are set as 400 . The parameters range are listed below: $N_{p} \in[3,6], t_{s} \in[5,10] \mathrm{mm}, t_{m} \in[5,10] \mathrm{mm}$, $t_{h} \in[0.2,1] \times t_{m} \mathrm{~mm}, d_{c} \in[0.3,0.8] \mathrm{mm}$, and $L_{w}=[2,6]$. The obtained Pareto front is shown in Fig. 10. The conflicting nature of the objectives is evident from the final shape of the front. Most of the solutions in Pareto front are in the 4-pole design which indicates it is the best choice for the $\pm 10^{\circ}$ working range. The final selected design chooses a solution with a satisfactory dynamic response time $(7 \mathrm{~ms})$, torque linearity characteristic $\left(\Delta K_{t}^{\max }<0.02 \mathrm{Nm}\right)$, and the torque constant $(0.69 \mathrm{Nm} / \mathrm{A})$. The final rounded parameters of the selected solution are: $N_{p}=4, t_{r}=7.4, t_{m}=8.2$, $t_{s}=7.9, t_{h}=5, d_{c}=0.5, L_{w}=4$.

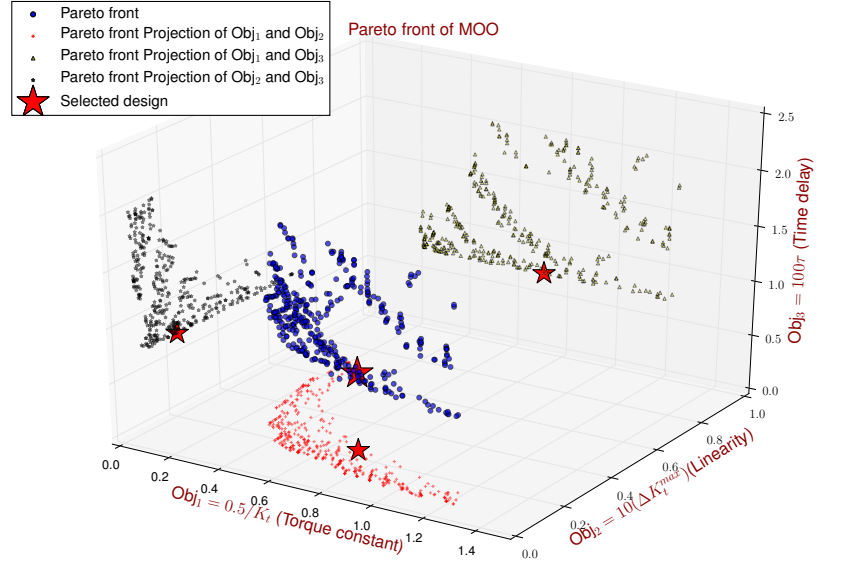

Fig. 10. The 3-D scatter and 2-D projections of the Pareto front designs

\section{E. Further optimization of rotor}

The Fig. 6 indicates that the flux density distribution in the rotor is not uniform. It is very low where close to the middle of the radial-magnetized PM where is colored with blue and green. The material in this region is hardly used, resulting in additional idle rotational inertia and hence degradation in dynamic response. Therefore it can be further optimized. The final optimized shape of the rotor is shown in Fig. 11(a). The material where $B$ is very low is removed. The flux density contour map indicates the flux density distribution of rotor is more uniform than the original design. The flux density in airgap before and after the removal of material of the rotor are shown in Fig. 11(b). It indicates that the reduction is negligible and thus the torque will keep the same. But the reduce of moment of inertia by $7 \%$ improves the system dynamics.

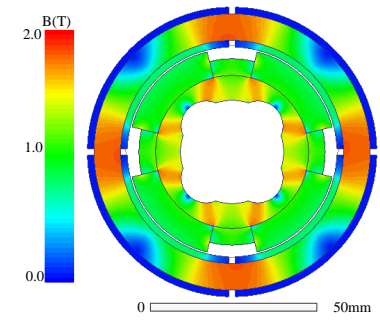

(a) Flux density distribution after remove extra rotor material.

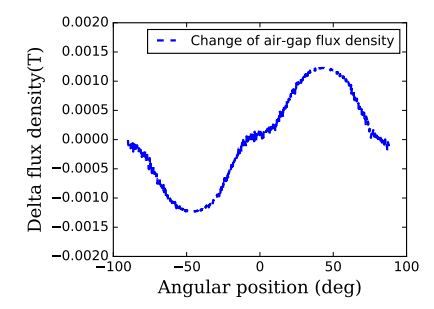

(b) The change of air-gap flux density after cut extra material.
Fig. 11. Magnetic field change due to removal of rotor extra material

\section{PRototype AND EXPERIMENT RESUltS}

A prototype based on the optimized parameters was manufactured as shown in Fig. 12. A redundancy design approach is adopted for fault tolerance requirements. The rotor and the stator are assembled in the housing and installed onto the test bench. A magnetic meter is used to measure the air-gap flux density, and a torque sensor to measure the output torque. The dynamic performance is tested by a step response of position close loop control. A servo amplifier which has current close loop (GLENTEK SMA5005 H Bridge Linear servo amplifier) is used to provide the current. A rotary encoder (Heidenhein 
ROD 1080 3600) is used to measure the angular position of the rotor. A real-time industrial computer is used to sample the position and achieve the close loop control.

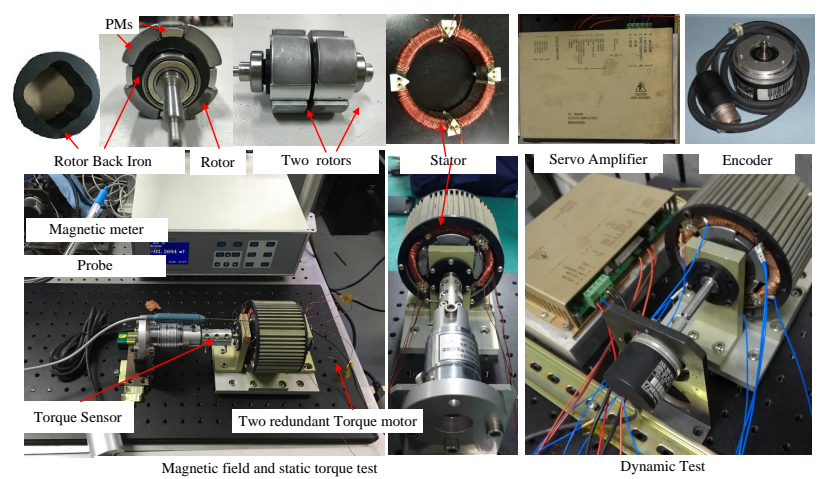

Fig. 12. Prototype of motor and test setup

With this setup, when the rotor is suspended in the neutral position, the flux density along the air-gap is tested and is shown in Fig. 13. It is lightly smaller than the FEA result. This can be attributed to the PMs being smaller than the actual designed size because of manufacture and assembly tolerances.

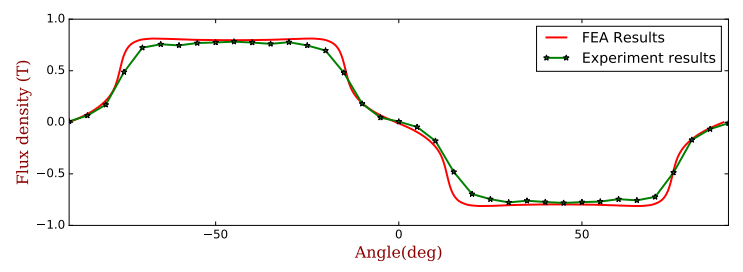

Fig. 13. Measured air-gap flux density characteristics

The torque constant is measured by the torque sensor when the rotor at different positions with a unit input current. The back-EMF coefficient is obtained by dividing the generated voltage of coil to velocity when the rotor is rotated by another motor. The torque constant is shown in Fig. 14(a), and the back-EMF is shown in Fig. 14(b). The results indicate that the torque is highly linear with the current and the characteristics are almost the same in the range of $\pm 10^{\circ}$. The results also show a discrepancy of about $8 \%$ between the FEA result and the measurement which can be due to the smaller air-gap flux density in practice, and the winding turns are less than the design value.

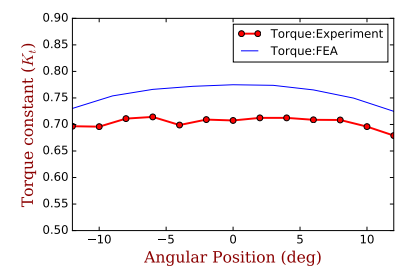

(a) Torque constant

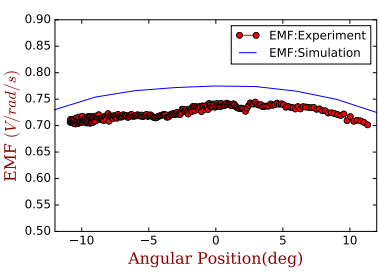

(b) Back-EMF
Fig. 14. Measured torque characteristics.

The dynamic performance is assessed by closed loop step response. The amplitude of step is $5^{\circ}$. The results are shown in Fig. 15. The simulation results before and after the removal of the extra material of the rotor indicate that it improves the dynamic performance due to reduction of inertia. The best simulation performance indicates the rise time is less than $8 \mathrm{~ms}$. The experimental result is slower than that of the simulation, but still less than $10 \mathrm{~ms}$ which satisfies the servo control requirement.

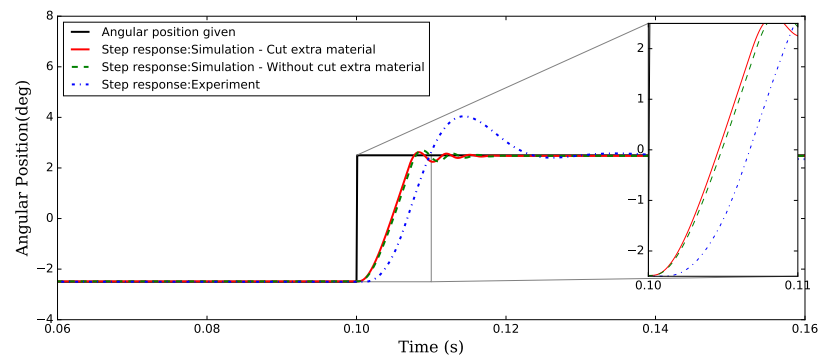

Fig. 15. Position closed loop step response

\section{CONCLUSION}

The design and optimization of a small range LATM for the rotary servo valve applications has been proposed. The proposed LATM uses slot-less toroidally wound stator, and a PM-mounted rotor structure which helps to achieve a high torque density and uniform flux density along the air-gap, and eliminates torque ripple in the working range, making it particularly suitable for high precision limited range position servo control. The use of Halbach array increase torque-toinertia ratio and reduction in core losses. An analytical model was derived based on the EMC method for preliminary design phase. The proposed EMC model was fully parameterized and arranged in KCL matrix format which is ease to extend. An iterating technique to obtain an accurate permeability of the nonlinear magnetic material is also incorporated into the model. The Pareto front, which consider the performances of torque constant, torque linearity, and dynamic response time, was obtained by MOPSO method. Finally, a balance solution in the Pareto front was selected and validated by the FEA method. The rotary inertia of rotor is optimized by removing idle parts that carry negligible flux. The experimental results on a prototype verified the design and optimization strategy very well. Good transient response and high linearly in small working range make the proposed structure very acceptable for limited angle servo control application.

\section{APPENDIX A \\ DETAILS OF EMC MODEL}

The sub-matrices in matrix $G$ are given in the following equations. All the subscripts are according to Fig. 4 for easy to understand. The subscripts can be extended easily if more sectors are divided.

$$
\boldsymbol{G}_{11}=\left[\begin{array}{ccccc}
\frac{1}{R_{1,1}^{0,1}} & \frac{-1}{R_{1,1}^{1,2}} & 0 & \cdots & 0 \\
\frac{-1}{R_{1,1}^{0,1}} & G_{11}^{2,2} & \frac{-1}{R_{1,1}^{2,3}} & \cdots & 0 \\
\vdots & & & \ddots & 0 \\
0 & \cdots & -\frac{1}{R_{1,1}^{6}} & G_{11}^{7,7} & \frac{-1}{R_{1,1}^{6,7}} \\
0 & \cdots & 0 & \frac{-1}{R_{1,1}^{5,6}} & \frac{1}{R_{1,1}^{6,7}}
\end{array}\right]_{8 \times 8}
$$




$$
\begin{aligned}
& G_{11}^{2,2}=\frac{1}{R_{1,1}^{0,1}}+\frac{1}{R_{1,2}^{0,1}}+\frac{2}{R_{1,1}^{1,2}}, G_{11}^{7,7}=\frac{1}{R_{1,1}^{5,6}}+\frac{1}{R_{1,2}^{6,7}}+\frac{1}{R_{1,1}^{6,7}} \\
& \boldsymbol{G}_{22}=\left[\begin{array}{ccccc}
\frac{1}{R_{2,3}^{0,0}} & 0 & 0 & \cdots & 0 \\
0 & G_{22}^{22} & \frac{-1}{R_{2,2}^{1,2}} & \cdots & 0 \\
\vdots & & & \ddots & 0 \\
0 & \cdots & \frac{-1}{R_{2,2}^{5,6}} & G_{22}^{77} & 0 \\
0 & \cdots & 0 & 0 & G_{22}^{88}
\end{array}\right]_{8 \times 8} \\
& G_{22}^{22}=\frac{1}{R_{1,2}^{1}}+\frac{1}{R_{2,3}^{2}}+\frac{1}{R_{2,2}^{2}}, G_{22}^{77}=\frac{1}{R_{1,2}^{6}}+\frac{1}{R_{2,2}^{6}}+\frac{1}{R_{2,3}^{6}} \\
& G_{22}^{88}=\frac{1}{R_{1,2}^{N+1}}+\frac{1}{R_{2,3}^{N+1}} \\
& \boldsymbol{G}_{33}=\left[\begin{array}{ccccc}
G_{33}^{11} & 0 & 0 & \cdots & 0 \\
0 & G_{33}^{22} & \frac{-1}{R_{3,3}^{1,2}} & \cdots & 0 \\
\vdots & & & \ddots & \frac{-1}{R_{3,3}^{4,5}} \\
0 & \cdots & \frac{-1}{R_{3,3}^{4,5}} & G_{33}^{66} & 0 \\
0 & \cdots & 0 & 0 & G_{33}^{77}
\end{array}\right]_{7 \times 7} \\
& G_{33}^{11}=\frac{1}{R_{2,3}^{0,0}}+\frac{1}{R_{3,4}^{0,0}}, G_{33}^{22}=\frac{1}{R_{2,3}^{1,1}}+\frac{1}{R_{2,3}^{1,2}}+\frac{1}{R_{3,3}^{1,2}}+\frac{1}{R_{3,4}^{1,1}} \\
& G_{33}^{66}=\frac{1,3}{R_{3,3}^{4,5}}+\frac{1}{R_{2,3}^{5,5}}+\frac{1}{R_{2,3}^{5,6}}, G_{33}^{77}=\frac{1,3}{R_{2,3}^{6,7}}+\frac{1}{R_{3,4}^{6,6}} \\
& \boldsymbol{G}_{44}=\left[\begin{array}{ccccc}
G_{44}^{11} & 0 & 0 & \cdots & 0 \\
0 & G_{44}^{22} & 0 & \cdots & 0 \\
\vdots & & & \ddots & 0 \\
0 & \cdots & 0 & G_{44}^{66} & 0 \\
0 & \cdots & 0 & 0 & \frac{1}{R_{3,4}^{6,6}}+\frac{1}{R_{4,5}^{6,6}}
\end{array}\right]_{7 \times 7} \\
& G_{44}^{11}=\frac{1}{R_{3,4}^{0,0}}+\frac{1}{R_{4,5}^{0,1}}, G_{44}^{22}=\frac{1}{R_{3,4}^{1,1}}+\frac{1}{R_{4,5}^{1,2}}, G_{44}^{66}=\frac{1}{R_{3,4}^{5,5}}+\frac{1}{R_{4,5}^{5,5}} \\
& \boldsymbol{G}_{55}=\left[\begin{array}{ccccc}
\frac{1}{R_{5}^{0,1}} & \frac{-1}{R_{5,5}^{0,1}} & 0 & \cdots & 0 \\
\frac{-1}{R_{5,5}^{0,1}} & G_{55}^{22} & \frac{-1}{R_{5,5}^{1,2}} & \cdots & 0 \\
\vdots & & & \ddots & 0 \\
0 & \cdots & \frac{-1}{R_{5,5}^{5,6}} & G_{55}^{77} & \frac{-1}{R_{5,5}^{6,7}} \\
0 & \cdots & 0 & \frac{-1}{R_{5,5}^{6,7}} & \frac{1}{R_{5,5}^{6,7}}
\end{array}\right]_{8 \times 8} \\
& G_{55}^{22}=\frac{1}{R_{5,5}^{0,1}}+\frac{1}{R_{4,5}^{0,1}}+\frac{1}{R_{5,5}^{1,2}}, G_{55}^{77}=\frac{1}{R_{5,5}^{5,6}}+\frac{1}{R_{4,5}^{6,6}}+\frac{1}{R_{5,5}^{6,7}} \\
& \boldsymbol{G}_{12}=\boldsymbol{G}_{21}^{T}\left[\begin{array}{cccc}
0 & \cdots & 0 & 0 \\
\frac{-1}{R_{1,2}^{0,1}} & 0 & \cdots & 0 \\
\vdots & \cdots & \ddots & 0 \\
0 & 0 & 0 & \frac{-1}{R_{1,2}^{6,7}} \\
0 & \cdots & 0 & 0
\end{array}\right]_{8 \times 8} \\
& \boldsymbol{G}_{23}=\boldsymbol{G}_{32}^{T}\left[\begin{array}{cccc}
\frac{-1}{R_{2,3}^{0,0}} & 0 & \cdots & 0 \\
\vdots & 0 & \ddots & 0 \\
0 & 0 & \frac{-1}{R_{2,3}^{5,6}} & 0 \\
0 & \cdots & 0 & \frac{-1}{R_{2,3}^{6,7}}
\end{array}\right]_{8 \times 7} \\
& \boldsymbol{G}_{34}=\boldsymbol{G}_{43}^{T}\left[\begin{array}{cccc}
\frac{-1}{R_{3,4}^{0,0}} & 0 & \cdots & 0 \\
0 & \vdots & \ddots & 0 \\
0 & 0 & \frac{-1}{R_{3,4}^{5,5}} & 0 \\
0 & \cdots & 0 & \frac{-1}{R_{3,4}^{6,6}}
\end{array}\right]_{7 \times 7}
\end{aligned}
$$

$$
\boldsymbol{G}_{45}=\boldsymbol{G}_{54}^{T}\left[\begin{array}{cccccc}
0 & \frac{-1}{R_{4,5}^{0,1}} & 0 & 0 & \cdots & 0 \\
0 & 0 & \cdots & \ddots & 0 & 0 \\
0 & 0 & 0 & \frac{-1}{R_{4,5}^{4,5}} & 0 & 0 \\
0 & \cdots & 0 & 0 & \frac{-1}{R_{4,5}^{6,6}} & 0
\end{array}\right]_{7 \times 8}
$$

All the reluctances in the EMC model can be calculated based on the shapes of the flux tubes as listed in Table II [14]. All reluctances are calculated by $R=l / \mu s$, where $l$ and $s$ are the length and the cross-section area of flux path, respectively.

TABLE ॥

Calculation of Reluctance of EMC Model

\begin{tabular}{ccc}
\hline Reluctances & Shape & Equation \\
\hline$R_{1,1}^{p 1, p 2}, R_{2,2}^{p 1, p 2}, R_{2,3}^{p 1, p 2}$, & Cuboids & $R=\frac{T}{\mu W L}$ \\
$R_{3,4}^{p 1,22}, R_{5,5}^{p 1, p 2}$ & & Schematic \\
$R_{1,2}^{0,1}, R_{1,2}^{1,2}, R_{1,2}^{5,6}, R_{2,2}^{1,2}$, & Quarter & $R=\frac{\pi}{4 \mu L}$ \\
$R_{2,2}^{5,6}, R_{2,3}^{1,2}, R_{2,3}^{5,5}, R_{4,5}^{p 1, p 2}$ & Cylinder &
\end{tabular}

The equivalent flux source of the PMs are transformed from magneto-motive source which can be expressed as

$$
\phi_{2,3}^{n}=H_{c} t_{m}^{n} / R_{2,3}^{n} \quad n=1,2, \ldots, 6,7,
$$

where $H_{c}$ is coercive force of PM, $t_{m}^{n}$ the thickness of PM. The equivalent flux sources of th PM 3 can be expressed as

$$
\phi_{2,2}^{n}=H_{c} 0.5\left(r_{\text {ro }}+r_{\mathrm{h}}\right) \theta^{n} / R_{2,3}^{n} \quad n=3,4,5,
$$

The equivalent flux sources of winding current are given by

$$
\phi_{5,5}^{n}=N_{t}^{n} I / R_{5,5}^{n} \quad n=1,2, \ldots, 6,7,
$$

where $N_{t}^{n}$ is the winding turns in the $n$th sector.

Some notes and assumptions are as follows:

(1)For iterative calculation of the permeability, the input current should be set as 0 . Otherwise because the equivalent of flux source of winding will change the inner flux of the source, i.e. the flux density of stator, which will make the iteration not able to converge. After the permeability has converged, then fix the permeability and consider the current flux source to get the final flux. Since the flux generated by the current is relatively small, any error would be within an acceptable range.

(2)In order to keep the EMC model invariant when the rotor changes its position, the rotor is regarded as fixed and the coils are regarded as rotating on the stator. Then only need to change the winding turns in each sector for different angular position. It is much easier for programing and get same results.

\section{REFERENCES}

[1] C. Dawson and H. Bolton, "Limited motion rotary actuators of the toroidal-stator, permanent-magnet rotor type," in IEE Proceedings B (Electric Power Applications), vol. 129, no. 4. IET, 1982, pp. 190-198.

[2] C.-C. Tsai, S.-C. Lin, H.-C. Huang, and Y.-M. Cheng, "Design and control of a brushless DC limited-angle torque motor with its application to fuel control of small-scale gas turbine engines," Mechatronics, vol. 19, no. 1, pp. 29-41, 2009. 
[3] P. Hekmati and M. Mirsalim, "Design and analysis of a novel axialflux slotless limited-angle torque motor with trapezoidal cross section for the stator,' IEEE Transactions on Energy Conversion, vol. 28, DOI 10.1109/TEC.2013.2280248, no. 4, pp. 815-822, Dec. 2013.

[4] M. Roohnavazfar, M. Houshmand, R. N. Zarandi, and M. Mirsalim, "Optimization of design parameters of a limited angle torque motor using analytical hierarchy process and axiomatic design theory," Production \& Manufacturing Research, vol. 2, no. 1, pp. 400-414, 2014.

[5] R. Nasiri-Zarandi, M. Mirsalim, and A. Cavagnino, "Analysis, optimization, and prototyping of a brushless DC limited-angle torque-motor with segmented rotor pole tip structure," IEEE Transactions on Industrial Electronics, vol. 62, DOI 10.1109/TIE.2015.2402115, no. 8, pp. 49854993, Aug. 2015

[6] R. P. Praveen, M. H. Ravichandran, V. T. S. Achari, V. P. J. Raj, G. Madhu, and G. R. Bindu, "A novel slotless halbach-array permanentmagnet brushless DC motor for spacecraft applications," IEEE Transactions on Industrial Electronics, vol. 59, DOI 10.1109/TIE.2011.2161058, no. 9, pp. 3553-3560, Sep. 2012.

[7] S. Wu, Z. Jiao, L. Yan, R. Zhang, J. Yu, and C. Y. Chen, "Development of a direct-drive servo valve with high-frequency voice coil motor and advanced digital controller," IEEE/ASME Transactions on Mechatronics, vol. 19, DOI 10.1109/TMECH.2013.2264218, no. 3, pp. 932-942, Jun. 2014.

[8] K. Yamazaki and H. Ishigami, "Rotor-shape optimization of interior-permanent-magnet motors to reduce harmonic iron losses," IEEE Transactions on Industrial Electronics, vol. 57, DOI 10.1109/TIE.2009.2025285, no. 1, pp. 61-69, Jan. 2010.

[9] W. Fei, P. C. K. Luk, J. X. Shen, B. Xia, and Y. Wang, "Permanentmagnet flux-switching integrated starter generator with different rotor configurations for cogging torque and torque ripple mitigations," IEEE Transactions on Industry Applications, vol. 47, DOI 10.1109/TIA.2011.2125750, no. 3, pp. 1247-1256, May. 2011.

[10] Y. S. Chen, Z. Q. Zhu, and D. Howe, "Slotless brushless permanent magnet machines: influence of design parameters," IEEE Transactions on Energy Conversion, vol. 14, DOI 10.1109/60.790936, no. 3, pp. 686691, Sep. 1999.

[11] H. I. Lee and M. D. Noh, "Optimal design of radial-flux toroidally wound brushless DC machines," IEEE Transactions on Industrial Electronics, vol. 58, DOI 10.1109/TIE.2010.2054052, no. 2, pp. 444-449, Feb. 2011.

[12] K. Chau, M. Cheng, and C. Chan, "Nonlinear magnetic circuit analysis for a novel stator doubly fed doubly salient machine," IEEE Trans. Magn., vol. 38, no. 5, pp. 2382-2384, 2002.

[13] H. Polinder, J. G. Slootweg, M. J. Hoeijmakers, and J. C. Compter, "Modeling of a linear PM machine including magnetic saturation and end effects: Maximum force-to-current ratio," IEEE Trans. Ind. Appl., vol. 39, no. 6, pp. 1681-1688, 2003.

[14] Y. Liu, M. Zhang, Y. Zhu, J. Yang, and B. Chen, "Optimization of voice coil motor to enhance dynamic response based on an improved magnetic equivalent circuit model," IEEE Trans. Magn., vol. 47, no. 9, pp. 2247-2251, 2011.

[15] C. Pompermaier, K. Kalluf, A. Zambonetti, M. Luz, and I. Boldea, "Small linear pm oscillatory motor: magnetic circuit modeling corrected by axisymmetric 2-D FEM and experimental characterization," IEEE Trans. Ind. Electron., vol. 59, no. 3, pp. 1389-1396, 2012.

[16] M.-F. Hsieh and Y.-C. Hsu, "A generalized magnetic circuit modeling approach for design of surface permanent-magnet machines," IEEE Trans. Ind. Electron., vol. 59, no. 2, pp. 779-792, 2012.

[17] J. R. Brauer, Magnetic actuators and sensors. John Wiley \& Sons, 2006.

[18] B. Han, Q. Xu, and Q. Yuan, "Multiobjective optimization of a combined radial-axial magnetic bearing for magnetically suspended compressor," IEEE Transactions on Industrial Electronics, vol. 63, DOI 10.1109/TIE.2015.2509905, no. 4, pp. 2284-2293, Apr. 2016.

[19] D. K. Lim, K. P. Yi, S. Y. Jung, H. K. Jung, and J. S. Ro, “Optimal design of an interior permanent magnet synchronous motor by using a new surrogate-assisted multi-objective optimization," IEEE Transactions on Magnetics, vol. 51, DOI 10.1109/TMAG.2015.2449872, no. 11, pp. 1-4, Nov. 2015.

[20] F. Cupertino, G. Pellegrino, and C. Gerada, "Design of synchronous reluctance motors with multiobjective optimization algorithms," IEEE Trans. Ind. Appl., vol. 50, DOI 10.1109/TIA.2014.2312540, no. 6, pp. 3617-3627, Nov. 2014.

[21] H. Duan and L. Gan, "Orthogonal multiobjective chemical reaction optimization approach for the brushless DC motor design," IEEE Trans. Magn., vol. 51, no. 1, pp. 1-7, 2015.
[22] C. Coello, G. Pulido, and M. Lechuga, "Handling multiple objectives with particle swarm optimization," IEEE Trans. Evol. Comput., vol. 8, DOI 10.1109/TEVC.2004.826067, no. 3, pp. 256-279, Jun. 2004

[23] H. Abbass, R. Sarker, and C. Newton, "PDE: a pareto-frontier differential evolution approach for multi-objective optimization problems," in Evolutionary Computation, 2001. Proceedings of the 2001 Congress on, vol. 2, DOI 10.1109/CEC.2001.934295, 5 2001, pp. 971-978.

[24] K. DEB, "A fast elitist non-dominated sorting genetic algorithm for multi-objective optimization: NSGA-2," IEEE Trans. Evol. Comput., vol. 6 , no. 2, pp. 182-197, 2002. 